\title{
Indoor Navigation System of Faculty of Civil Engineering, ITU: A BIM Approach
}

\author{
Berk TARIHMEN, Benay DIYARBAKIRLI, Mustafa Onur KANBUR, \\ Hande DEMIREL \\ Istanbul Technical University, Faculty of Civil Engineering, Department of Geomatics \\ Engineering, Istanbul, Turkey

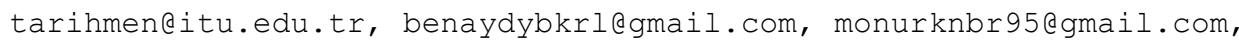

ORCID 0000-0002-3545-6118, ORCID 0000-0002-3790-9547, ORCID 0000-0001-5946-3999, ORCID 0000-0003-0338-791X

\begin{abstract}
This study aims to integrate BIM/GIS to detect indoor navigation routes, where these routes are simulated afterwards via connecting such information into a gaming environment. For testing these developed concepts, some sections of the Istanbul Technical University, Faculty of Civil Engineering building are modelled utilizing the BIM concepts. The designed methodology includes generating the BIM model, transforming the model into international standard Industry Foundation Classes (IFC) and again transforming this into FBX format to work in Unity platform that is a gaming environment. Furthermore, these processes are bi-directional. The final deliverable enables the user to navigate in the faculty building via a three-dimensional BIM-based environment. Via the described concept, easy, repeatable and reliable three-dimensional information is achieved that is the initial step for smart cities.
\end{abstract}

Keywords: BIM (Building Information Modelling), GIS (Geographic Information System), IFC, Indoor Navigation, Gaming Environment.

\section{Introduction}

The recent very popular term "Smart City" needs a digital city environment including buildings, roads, parks, infrastructure and several others, where the integration of Information and Communication Technology (ICT) and Internet of Things (IoT) technologies will be performed upon this fundamental/core basis. (Ma and Ren, 2017). The smart city has beneficial impacts on the cities that need to resolve urban expansion, have a newer infrastructure, renovate older buildings and make cities eco-friendly. The creation of smart city process includes using data by considering physical constituents of the city, environments around it and urban services that belong to the city. Different methodologies can be used for the creation of smart cities such as Geographic Information Systems (GIS) for urban infrastructures, Building Information Modelling 
(BIM) for building representation (Alileche et al., 2017). Hence, data acquisition/collection is the initial step for the creation of smart cities. After this process, collected data is managed and transformed with BIM and GIS technologies (Ma and Ren, 2017). Integration between these two technologies is significant for the creation of projects that are beneficial to apply smart city applications.

Building Information Modelling is a process of creating a 3D model of a structure that represents physical and semantic attributes of it. Apart from traditional ComputerAided Design (CAD) drawings, BIM approach provides more accurate and detailed information about the structure that is modelled. It is not only used for the design process but also contributes to the workflow of the project (Cheng et al., 2017). It provides collaboration between different disciplines, has rich semantic information and could integrate geometric and parametric attributes of a 3D model. However, this model is stand alone and not integrated within the city environment. The relationship between other building and environment is not foreseen. Furthermore, queries that will enhance this experience are limited. These limits to understand the full capacity of this digital environment. Such problems could be diminished by integrating the BIM and GIS, where it is also possible to perform spatial analysis for the building. (Dias et al., 2014). The indoor navigation and related scenario base analyses could be one of the most popular outputs of such integration.

Indoor navigation is a technological development that provides to locate people and shows the path to target destination at indoor locations (Xu et al., 2017). Indoor navigation is a necessity and almost a standard application for places such as airports, shopping malls to enlighten the complex task of navigating in complex and sometimes unknown environments. Hence, this will reduce time and complexity, where the traces could be used for business analytics and better management. In several projects, this process of navigation is supported via sensors, where the geometry and topology of the building need to the explored in detail. BIM contributes to generating indoor navigation system in two aspects, namely: a) BIM has the 3D coordinate information for georeferencing, b) provides topologic and geometric rules to generate navigation system for the building (Costin and Teizer, 2014).

The construction and automatization of indoor navigation are multifaceted and more detailed analyses are required to understand the nature of this complex system. The two above mentioned systems namely BIM and GIS could be beneficiary for this generation, however, there exit bottlenecks. First of all, BIM and GIS are originally created for different purposes. GIS technology is feasible to create a model of the real world, on the other hand BIM is developed for the contribution of architectural works (Cheng et al., 2017). Furthermore, data types, data models and used software for BIM and GIS are different (Brandolini et al., 2018). Hence, two different perspectives need to be integrated to generate indoor navigation system via BIM and GIS. Furthermore, the developed system requires well described semantic, functional and $3 \mathrm{D}$ information of elements and their spatial relationship within each other in the building (Isikdag et al., 2013). Additionally, the indoor navigation system depends on a specific coordinate system and requires high semantic information, which in return make the integration between BIM and GIS necessary, hence challenging.

The methods and approaches in Geomatics Engineering discipline contribute to the solution of above-mentioned problems in BIM/GIS integration and indoor navigation process in many aspects. Firstly, under the influence of developing technology, data collection methods in Geomatics Engineering discipline such as laser scanning or other point clouds detection technology such as photogrammetry can be an option to gather 
highly accurate 3D spatial data (Behan, 2013) that is the primary step for the establishing the BIM model. Secondly, during the modelling process, this generated spatial data plays an important role to create a more detailed and semantically rich model. Furthermore, using GIS technology BIM model can be georeferenced. Additionally, algorithms that belong to GIS such as shortest path, impact area, the sight of view, visualization and many others could be useful for producing an indoor navigation system and testing scenario-based analyses.

Within the scope of this study, the integration of BIM/GIS is performed to detect indoor navigation routes, where these routes are simulated afterwards via connecting such information into a gaming environment. For testing these developed concepts, some sections of the Istanbul Technical University, Faculty of Civil Engineering building are modelled in BIM. The developed concepts are successfully implemented and it is possible to move and navigate in this 3 dimensional BIM/GIS-based gaming environment. The remaining sections of this manuscript are organized as follows; the second section introduces the related work on indoor navigation, where the third section is on data used and methodology. The fourth section briefly illustrated the achieved results and the manuscript is finalized with the conclusion section.

\section{Related Works on Indoor Navigation}

Human beings are spending $80 \%-90 \%$ of their time indoors such as homes, offices, libraries, shopping malls, airports, where solutions getting attraction for indoor navigation are becoming popular due to recent developments in mobile phone innovations. (EPA, 1989; Zeinalipour-Yazti, 2017) Furthermore, buildings are getting complex that leads to an increased demand for indoor navigation. Deneux et al. (2020) have developed a system called BiMov that is used for path planning automatically in complex buildings. Path planning structure of BiMov has been represented as a graph and has three levels of detail: Macro graph has been created to identify topological relationships in the interior part of the building and has been provided a connection between spaces in the structure. The external graph has been built to define feasible route by considering structural obstacles like walls and columns in the building. The internal graph has been created to avoid obstacles that are inside of the building. The system extracts elements that are necessary for navigation purposes automatically and define the shortest path between spaces in the building via Dijkstra Algorithm (Deneux et al., 2020).

Teo and Cho (2016) proposed a Multi-Purpose Geometric Network Model (MGNM) to connect the indoor and outdoor environment for navigation purposes such as emergency response and pedestrian walking route by considering BIM model. (Teo and Cho, 2016). The system consists of space that represents indoor environments, portals that shows the connection between objects in the building and route that defines the navigation network. MGNM has been applied to an engineering building at the campus of National Chiao Tung University (NCTU). Several parts of the building have been extracted to generate navigation routes. Footprints have been calculated for MGNM. Then, footprints have been converted to the medial axis and centroids. Finally, MGNM has been built by using three elements such as corridor footprints, space footprints, and centroids of opening elements (Teo and Cho, 2016). 
In (Culp et al., 2010) BIM - Game prototype has been developed to improve architectural visualization and education. BIM information has been transformed into the game environment by using Revit API and Crossover module that provides interoperability between BIM and the gaming environments. Two different kinds of navigation techniques have been used: Interactive and Intelligent navigation. The user can walk in the model independently or user can use the navigation network to walk (Culp et al., 2010).

The research of Gao et al. (2012) has represented indoor path planning by using IFC files instead of traditional methods that use $2 \mathrm{D}$ drawings. The project has three main stages. The first stage is extracting information from IFC file. IFC file format has a high ability to preserve semantic and geometric information of the structure. The coordinates and attributes of building elements and obstacles have been identified via extraction. At the second stage, building components have been decomposed into planar grids by using the extracted information in the first stage. The spatial information has been represented numerically for each grid. At the final stage, the algorithm has found the shortest path from the current position to destination target by using numerical values in each grid. The shortest path has been calculated with Fast Marching Method (Gao et al., 2012).

Lin and Lin (2018) have proposed an approach called 'i-GIT algorithm' to generate the path in buildings where it is necessary to create an indoor navigation system for human beings. Three campus buildings at Feng Chia University and Taiwan High-Speed Rail (THSR) Taichung Station has been chosen to evaluate the feasibility and accuracy of indoor paths generated by i-GIT. IFC file format is the main requirements for path generation. Besides, I-GIT algorithm provides advantages for the navigation process. The length of the path is close to its actual measurements. It connects floors in the building with stairs, ramps and elevators successfully. It reduces the number of nodes with polygon regularization (Lin and Lin, 2018). Other researchers also presented studies on path generation by considering obstacles. (Xu et al., 2017).

Isikdag et al. (2013) have listed some requirements about the creation of the indoor navigation system and BIM oriented model. These requirements are defining semantic attributes of the building components, physical and functional characteristics of each element, $3^{\text {rd }}$ dimension and spatial relationship between building components. Isikdag et al. have developed a BIM oriented model that supports navigation process in indoor environments concerning these requirements. The creation of this system has utilized from the integration of BIM and GIS (Isikdag et al., 2013). The transformation between data formats belonged to these two different disciplines is also the main scientific interest of this manuscript, where several concepts are also followed.

\section{Data and Methodology}

\subsection{Data}

With the context of this study, the available data sources for the Faculty of Civil Engineering of Istanbul Technical University are utilized. Two-dimensional ComputerAided Design (CAD) drawings are obtained as illustrated in Figure 1, where these drawings only represent some portion of the building. These drawings are used as a base map to produce the BIM model of the structure. Geometric characteristics of the elements in the building need to be well represented to identify various categories such 
as wall, column and others. Every object that belongs to the building is modelled concerning the metric characteristics of 2D CAD drawings.

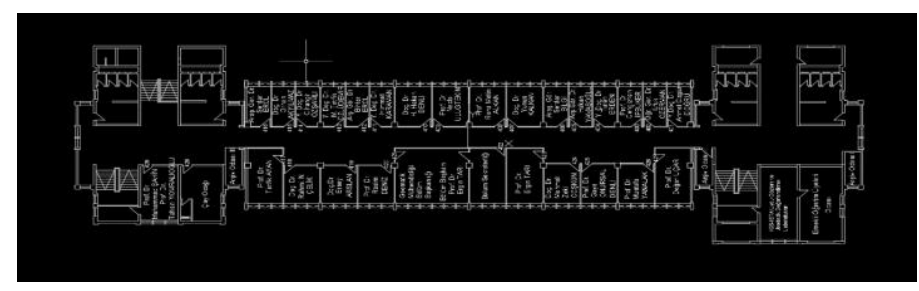

Figure 1: A Sample Floor Plan in two-dimension

\subsection{Methodology}

In order to generate a 3D BIM-based system for navigation purposes in the Faculty of Civil Engineering, Istanbul Technical University, two dimensional CAD plans are imported to the Autodesk Revit software. The aim is to produce a 3D model, where the navigation maps could be retrieved. All structural elements and components in the building are modelled by considering their geometric characteristics and material information. The model is exported as the IFC file to provide interoperability. IFC file includes all semantic and geometric information of the structure. After exporting the file, the location of the building is controlled by transforming the IFC file to Google Earth platform for future works. By using the SAFE/FME transformation platform, navigation routes in GIS environment are achieved. Then, the IFC file is converted to FBX format by keeping its geometry. Afterwards, the 3D model in FBX file format is imported into Unity software to define 3D topology between components of the structure. Hence, to generate simulations of navigation, the 3D model is associated with the gaming environment. So, navigable and non-navigable areas are defined via Unity software that is the gaming environment used. Then, first-person character is added to the model to represent the navigation process in the human eye. Finally, the 3D model created with BIM approach is ready to be used as a faculty information model and simulations for various profiles such as new-comers, evacuation scenario and detection of the impact area of sensors could be performed. The general workflow of the project is illustrated in Figure 2. 


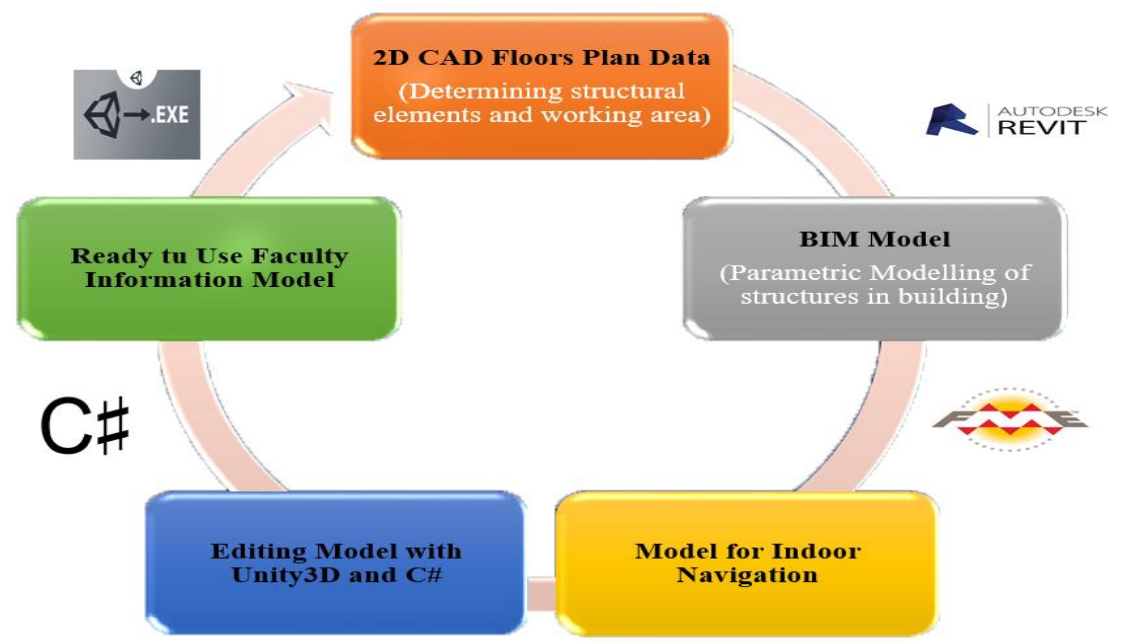

Figure 2. General Workflow of the Project

\section{Results and Discussion}

\subsection{Model Generation}

After obtaining floor plans that belong to the building, the parametric modelling process has been carried out. Two-dimensional CAD drawings are uses as an input to model the building according to the BIM procedure. The Autodesk Revit platform is used that enables users to produce three-dimensional models. Hence, the geometric and semantic characteristics of the structure are represented.

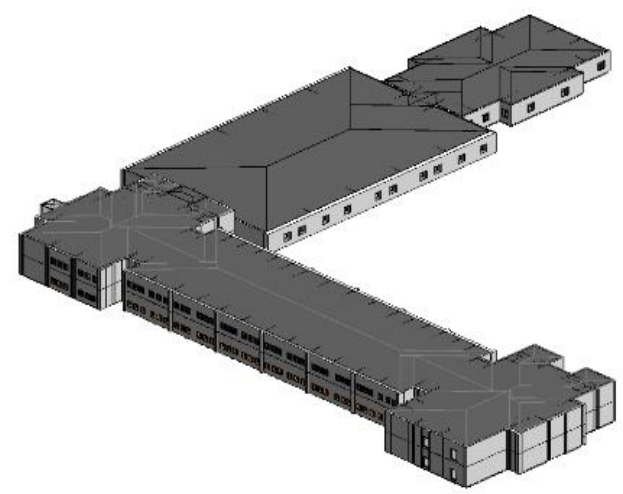

Figure 3. The Generated BIM of the Selected Floors

The following steps are followed to generate the 3D building. Firstly, height information of each floor is imported into the Revit software. It is significant to determine the base level and top level of components of the structure like walls, columns, stairs and others. Three floor plans are processed in the Revit software 
separately by considering their unit and height information. Using two-dimensional CAD plans, now it is possible to take measurements and to represent the geometry of elements. Columns and walls are created with respect to their thickness and depth level. Then, stairs, ground floors and doors are added to the model. Finally, ceilings and roofs are placed in the model. Components of the building are demonstrated in three dimensional (3D) parametric model, as presented in Figure 3.

\subsection{Model Transformation and Location Checking}

Then, coordinate values of centre of the building are adjusted and determined in Revit. The control process of the location of the building is carried out within the Safe/FME software. The parametric model is exported as IFC file that is a standard to keep geometric and semantic attributes of the model during the transformation process. IFC file format is transformed into .kml file format to control in Google Earth platform. The coordinates are inserted in WGS84 datum. The implemented transformation procedure in FME is illustrated below in Figure 4, where the parametric model in Google Earth Platform is illustrated in Figure 5. Hence, the integration of various systems is achieved.

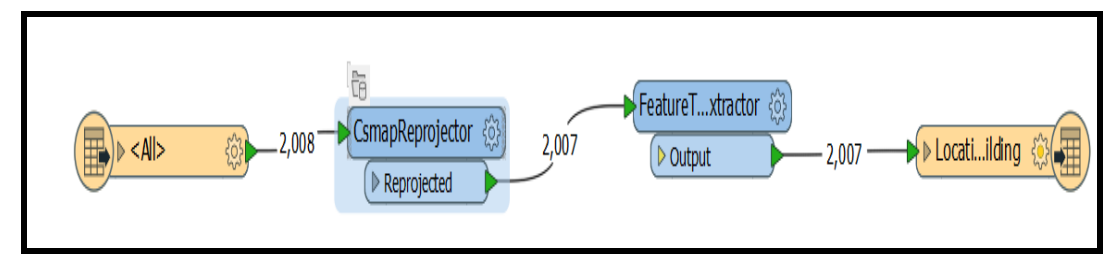

Figure 4. Transformation Modules Used in FME to Convert IFC to KML

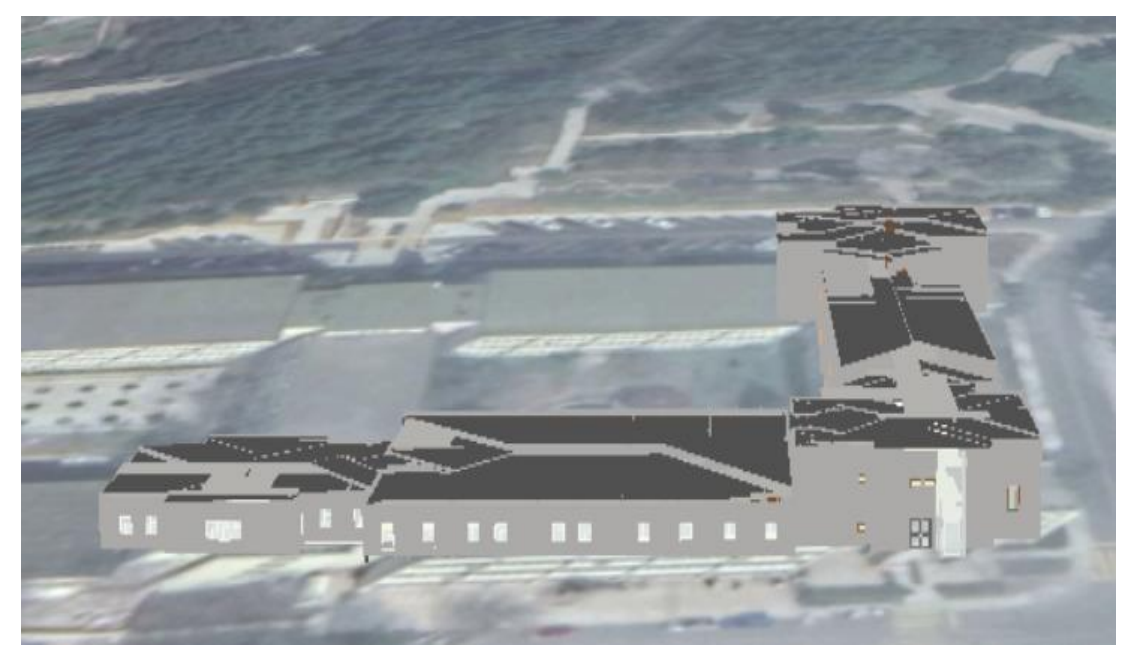

Figure 5. The Parametric Model in Google Earth Platform 
After this process, the IFC file format is transformed into the fbx file format to use the three dimensional model in the gaming environment, Unity platform. For this process, FME and its transformers are utilized, where the work-flow is illustrated in Figure 6.

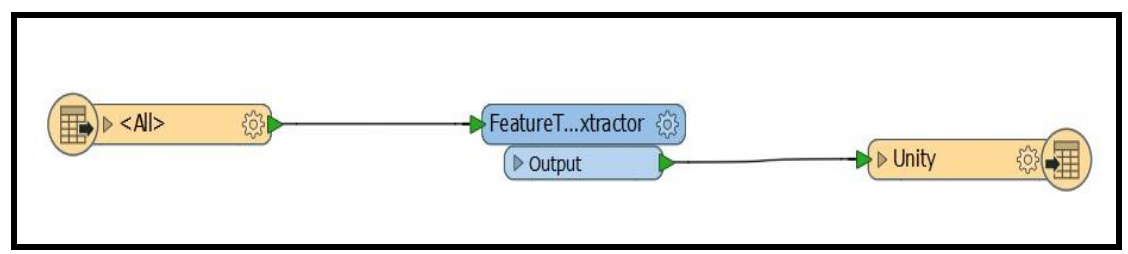

Figure 6. Transformation Modules Used in FME to Convert IFC to FBX

\subsection{Indoor Navigation in Unity}

The purpose of this step is to provide user to move in building spaces by defining navigable and non-navigable areas in the building by creating a virtual person character. Unity software is used for this step due to having high visual capability, flexibility and providing movement ability in the building. After transformation, there was some lack of visual representation and size of the structure. The transformed model was a bit darker than the original one and the size of the structure is quite larger than the original one. Firstly, the visual properties of the model are corrected by changing the colour of materials and textures of some physical objects such as doors, walls, windows, stairs, elevator and floors as shown in Figure 7. Then, the model is adjusted to represent its actual size via scaling in Unity. After the adjustment process, firstly, the spatial relationship between objects is described. The navigable and non-navigable areas are defined. Walls and columns are defined as non-navigable areas and ground floors and stairs are defined as navigable areas by adding mesh collider. Also, doors are specifically described whether they can be open or not by C\# scripting.

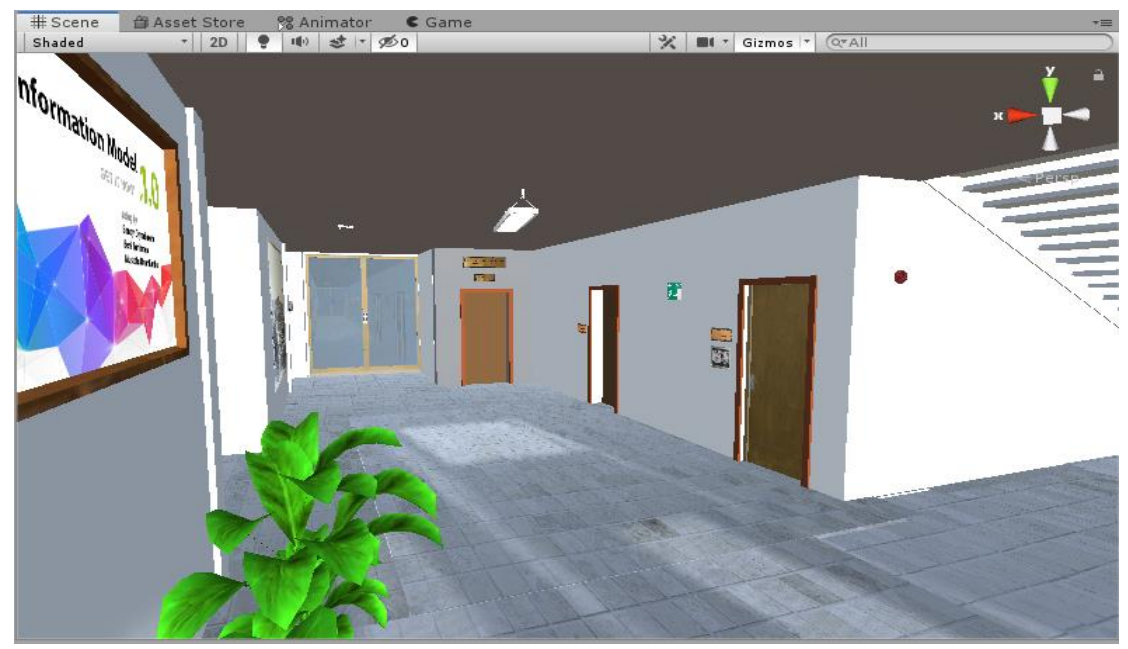

Figure 7. 3D Section of a Part of the Building in Unity 
Then, a first-person character that has a specific sight view like a human being is located in the 3-D building model. To provide character to move, the position of the first-person character is located in the model. Some attributes are added to the firstperson character such as walking speed, sight altitude via C\# programming language. Then boards that show owners of the offices and some furniture in the building are added to the model. Finally, the model is suitable to be used as a faculty information model that represents the location of indoor spaces and architectural characteristics of the building. Users are can move in the BIM-based 3D Model of the Faculty independently.

\subsection{Results and Discussion}

The system is successfully developed for the Faculty of Civil Engineering, Istanbul Technical University. The system shows that the BIM model can be functional in virtual environments for navigation purposes, where it could also be integrated with the gaming environment. Especially, using a character that has first-person control trait provides the user to move in the 3D model in the human eye.

The system is mainly developed to contribute to the orientation process of new students in the Faculty of Civil Engineering, ITU. As shown in Figure 8, the user can move on navigable areas independent from any path by considering $3 \mathrm{D}$ topology in the faculty. So, the user could be informed about the location of rooms and characteristics of the structure before going there in real life.

The system developed as a prototype to demonstrate that the navigation process is possible by representing high visual information of indoor spaces. As a result, the system requires some further works to test usability and if necessary improve its efficiency. Furthermore, the work-flow that is currently technically challenging could be automated. Additionally, the system will be tested in many aspects such as usability, user satisfaction and query time.

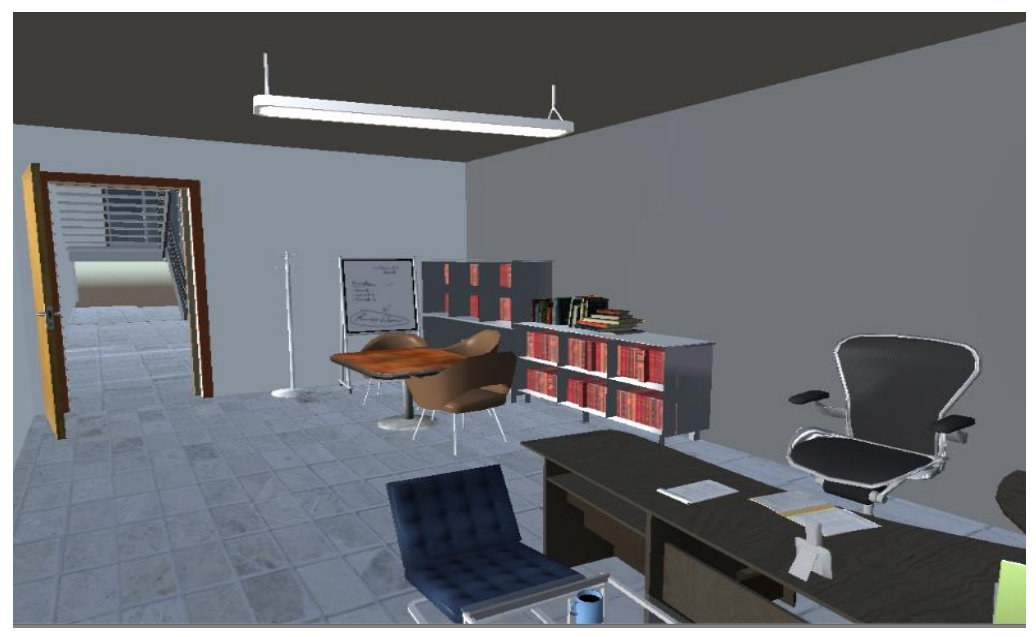

Figure 8. A Section of Lecturer's Room in the Model 


\section{Conclusion}

The generated indoor navigation model in the gaming environment enables users to have information about the location and attributes of places in the building. Firstly, a 3D parametric model is created from 2D CAD drawings that include three floors of the building. Then, this model is used to create a navigation system that demonstrates the location of rooms and includes physical objects and attributes of them. Additionally, the designed system is a BIM-based indoor navigation system can help people to find the briefest and quickest route in a complex structure.

The model can be used as a faculty information model to give information about the places in the faculty for the new-comers during the orientation phase. The model is compliant with current 3D standards, hence it is open to be improved via using programming languages. Besides, with the multi-strategy approach, wireless points or other types of sensors can be integrated into the model for determining current position automatically in complex structures, where this would be a step forward to "smart cities".

\section{References}

Alileche, L., Al Furjani, A., Shahrour, I., (2017), "Smart Cities: System and Tools used for the digital Modelling of Physical Urban Systems”, 2017 Sensors Networks Smart and Emerging Technologies (SENSET), Beirut, 2017, pp. 1-4, doi: 10.1109/SENSET.2017.8125056 .

Behan A., (2013). "Update on the BIM Education of Geomatics Surveyors", CITA BIM Gathering, 14th-15th November, 2013, Dublin Ireland, pp. 2-3.

Brandolini, M., Pili, D., Quaquero, E., Vacca, G., (2018), "Integrating BIM and GIS data to support the management of large building stocks", Int. Arch. Photogramm. Remote Sens. Spatial Inf. Sci., XLII-4, 647-653, https://doi.org/10.5194/isprs-archives-XLII-4-647-2018 .

Cheng, J., Li, X., Liu R., Liu, X., Wang X., Wright, G., (2017), "A State-of-the-Art Review on the Integration of Building Information Modeling (BIM) and Geographic Information System (GIS)." ISPRS Int. J. Geo-Inf. 6, no. 2: 53, DOI:10.3390/ijgi6020053.

Costin, A. and Teizer, J., (2014). "Utilizing BIM for Real-Time Visualization and Indoor Localization of Resources", 2014 International Conference on Computing in Civil and Building Engineering, pp. 650, DOI:10.1061/9780784413616.081

Culp, C., Graf, R., Yan, W., (2010), "Integrating BIM and gaming for real-time interactive architectural visualization", Automation in Construction, Issue 4, 2011, Pages 446-458, ISSN 0926-5805, https://doi.org/10.1016/j.autcon.2010.11.013

Deneux, D., Hamieh, A., Louhichi, B., Makhlouf, A., (2020), "A BIM-based method to plan indoor paths", Automation in Construction, Volume 113, 2020, 103120, ISSN 0926-5805, https://doi.org/10.1016/j.autcon.2020.103120

Dias, E., Fruijtier, S., Rafiee, A., Scholtend, H., (2014), "From BIM to Geo-analysis: View Coverage and Shadow Analysis by BIM/GIS Integration", Procedia Environmental Sciences, Volume 22, 2014, Pages 397-402, ISSN 1878-0296, https://doi.org/10.1016/j.proenv.2014.11.037. 
Gao, G., Gu, M., Han, X., Lai, C., Liu, S., Lin, Y., (2012), “The IFC-based path planning for 3D indoor spaces", Advanced Engineering Informatics, Volume 27, Issue 2, 2013, Pages 189-205, ISSN 1474-0346, https://doi.org/10.1016/j.aei.2012.10.001

Isikdag, U., Underwood, J., Zlatanova, S., (2013), “A BIM-Oriented Model for supporting indoor navigation requirements", Computers, Environment and Urban Systems, Volume 41, 2013, Pages112-123, ISSN 0198-9715, https://doi.org/10.1016/j.compenvurbsys.2013.05.001

Lin, P., Lin, Y., (2018), "Intelligent generation of indoor topology (i-GIT) for human indoor pathfinding based on IFC models and 3D GIS technology", Volume 94, 2018, Pages 340-359, ISSN 0926-5805, https://doi.org/10.1016/j.autcon.2018.07.016

Ma, Z., Ren, Y., (2017), "Integrated Application of BIM and GIS: An Overview", Volume 196, 2017, Pages 1072-1079, ISSN 1877-7058, https://doi.org/10.1016/j.proeng.2017.08.064.

Teo, T., Cho, K., (2016), "BIM-oriented indoor network model for indoor and outdoor combined route planning", Advanced Engineering Informatics, Volume 30, Issue 3, 2016, Pages 268282, ISSN 1474-0346, https://doi.org/10.1016/j.aei.2016.04.007

United States Environmental Protection Agency, (1989), “Report to Congress on indoor air quality”, Volume 2: Assessment and Control of Indoor Air Pollution, Indoor Air Division Office of Atmospheric and Indoor Air Programs Office of Air and Radiation and United States Environmental Protection Agency, Washington D.C.

Xu, M, Wei, S., Zlatanova, S., and Zhang, R., (2017). "Bim-Based Indoor Path Planning Considering Obstacles”, ISPRS Ann. Photogramm. Remote Sens. Spatial Inf. Sci., IV-2/W4, 417-423, https://doi.org/10.5194/isprs-annals-IV-2-W4-417-2017

Zeinalipour-Yazti, D., (2017), "Indoor Navigtion Services from Mobile Data", Advance in databases and Informatin sytems: $21^{\text {st }}$ European Conferece ADBIS 2017, Nicosia, Cyprus, September 24-27, Proceedings, Ed: Kirikova, M., Nørvåg, K., Papadopoulos, G.A, Springer, Sep 15, 2017 - Computers - 424 pages.

Received June 7, 2020, accepted June 8, 2020 as a paper from ISAG2019 